\title{
Case of Fournier's Gangrene in a Patient with Long-Term Indwelling Catheterization due to Urinary Incontinence after Open Radical Prostatectomy
}

\author{
Kyung Kgi Park, Sung Dae Kim, Young-Joo Kim, Jung-Sik Huh \\ Department of Urology, Jeju National University Hospital, Jeju National University, Graduate School of Medicine, Jeju, Korea
}

Fournier's gangrene is a life-threatening disease that needs to be treated as soon as possible. An 82-year-old male, who exchanged a urethral catheter once a month for urinary incontinence management after open radical prostatectomy, presented with an acute onset of mental change and general weakness. After ten days' hospitalization, the disease was diagnosed. The scrotal wall was opened, and the infectious tissue was exposed to the air and kept open with an aseptic dressing. After 45 days, his scrotal wound healed and returned to its typical appearance without scarring and wound disruption. He recovered fully from the infection. This paper reports a case of Fournier's gangrene in a patient with long-term indwelling catheterization due to urinary incontinence after an open radical prostatectomy with a literature review.

Keywords: Gangrene, Fournier's; Prostatectomy; Urinary incontinence; Catheters

Copyright $\subset$ c 2020, Korean Association of Urogenital Tract Infection and Inflammation. All rights reserved. (1) (\$) This is an open access article distributed under the terms of the Creative Commons Attribution (c) (1) (8) Non-Commercial License (http://creativecommons.org/licenses/by-nc/4.0) which permits unrestricted non-commercial use, distribution, and reproduction in any medium, provided the original work is properly cited.
Received: 16 April, 2020

Revised: 18 April, 2020

Accepted: 21 April, 2020

\author{
Correspondence to: Jung-Sik Huh \\ (iD) https://orcid.org/0000-0003-2767-4390 \\ Department of Urology, Jeju National University \\ Hospital, 15 Aran 13-gil, Jeju 63241, Korea \\ Tel: +82-64-717-1760, Fax: +82-64-717-1131 \\ E-mail: urohjs@jejunu.ac.kr
}

Fournier's gangrene is a life-threatening disease that needs to be treated as soon as possible. This disease is limited to the perineal and scrotal region of necrotizing fasciitis, and has high mortality if not treated appropriately. The causes of this disease include perineal abscess, chronic urethral catheterization, and bladder cancer, etc. Patients with diabetes are prone to this disease. Patients with three or more predisposing factors, which include diabetes mellitus, rectal and perianal abscess, hypertension, and urethral stricture, have the highest mortality rate [1].

As the interest in prostate disease increases, prostate cancer is often detected early, and a radical prostatectomy is often performed. On the other hand, the urethral sphincter could be weakened after the procedure [2], resulting in urinary incontinence is a complication after a radical prostatectomy. Most patients with urinary incontinence recover with time, but some patients have sustained symptoms. Su- stained urinary incontinence induces a poor quality of life for patients. The authors encountered an 82-year-old patient with urinary incontinence that did not recover after medication and Kegel exercise until seven years after an open radical prostatectomy. He had his urethral catheters exchanged once a month for the last two years. This paper reports a case of Fournier's gangrene in a patient with long-term indwelling catheterization due to urinary incontinence after an open radical prostatectomy with a review of the relevant literature.

\section{CASE REPORT}

An 82-year-old male presented at the emergency department with an acute onset of mental change and general weakness. He had previously undergone an open radical prostatectomy at another hospital due to prostate cancer 
seven years earlier. He did not have a prior medical history of diabetes mellitus and hypertension. His symptoms included fever, chills, and decreased blood pressure. He had urinary incontinence after surgery, with no improvement after antimuscarinics (solifenacin $10 \mathrm{mg}$, imidafenacin $0.1 \mathrm{mg}$ ), beta-3 adrenergic agonist (mirabegron $50 \mathrm{mg}$ ), and Kegel exercise over the previous seven years. Therefore, instead of taking medications, he had a urethral catheter exchanged once a month for the last two years. He was admitted to the department of internal medicine with the impression of pneumonia. The laboratory data revealed a serum level of white blood cells (WBCs) of $10.6 \times 10^{3} / \mu \mathrm{l}$, creatinine of $2.66 \mathrm{mg} / \mathrm{dl}$, C-reactive protein of $21.24 \mathrm{mg} / \mathrm{dl}$, and WBC and red blood cells of many/high power field in the urine. He received ciprofloxacin $500 \mathrm{mg}$ intravenous (IV) twice a day (bid) for four days, levofloxacin $500 \mathrm{mg}$ IV once a day (qd) for four days, and vancomycin $1 \mathrm{~g}$ and piperacillin/tazobactam 4.5 g IV three times a day (tid) for four days, consecutively. His general condition deteriorated gradually, and scrotal edema was detected after nine days. The laboratory data revealed a serum level of WBCs of $15.2 \times 10^{3} / \mu l$. The patient was then transferred to the department of urology for treatment. Computer tomography of the abdomen and pelvis was conducted, which revealed the presence of air formation over the scrotum and perineum (Fig. 1).

Suprapubic cystostomy was performed to divert urinary drainage instead of a urethra catheter. In addition, to enhance aeration, an incision was made, and aggressive surgical debridement on the scrotum and wound culture was performed under local anesthesia (Fig. 2). The daily aggres- sive aseptic dressing was required to improve his wound condition. The initial wound and blood culture revealed Proteus mirabilis and Enterococcus faecalis growth (Table 1). Two additional debridement procedures were done under monitored anesthetic care in the operation room. Two weeks after the surgery, his WBC count decreased gradually to $8.6 \times 10^{3} / \mu 1$, and his increased body temperature was normalized seven days after the operation.

His open scrotal wound was finally healed, and the cultures showed negative results at 22 days after the scrotal incision. The surgical wound was closed without an additional skin graft at postoperative day 25. After being transferred to the Department of Urology, ampicillin/sulbactam 3,000 mg q $6 \mathrm{hr}$ was prescribed for 28 days before closing the open surgical wound. After the reconstruction of the scrotum, cefoxitin $1 \mathrm{~g}$ tid was used until he was discharged from the hospital. The patient was discharged with full recovery after 45 days of admissions. After the discharge, a suprapubic cystostomy catheter was kept to resolve his urinary incontinence.

\section{DISCUSSION}

The best treatment for localized prostate cancer is a radical prostatectomy. Generally, the complications of radical prostatectomy were male sexual dysfunction and urinary incontinence [3]. The degree of urinary incontinence may vary according to the preservation of periurethral tissue [4]. Patients who have undergone a radical prostatectomy should perform pelvic floor muscle exercises or pelvic floor muscle
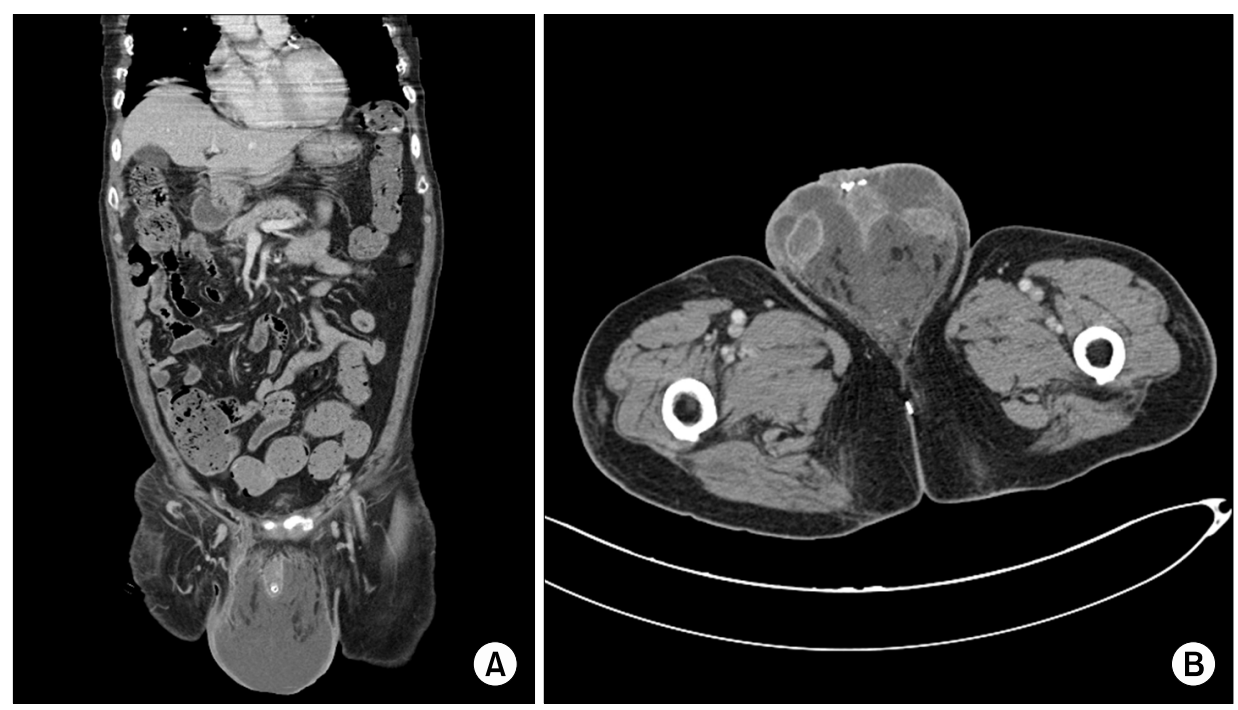

Fig. 1. (A, B) Computer tomography of the scrotum showing the presence of diffuse swelling of both scrotal wall and perineum with impending fluid collection or abscess. 

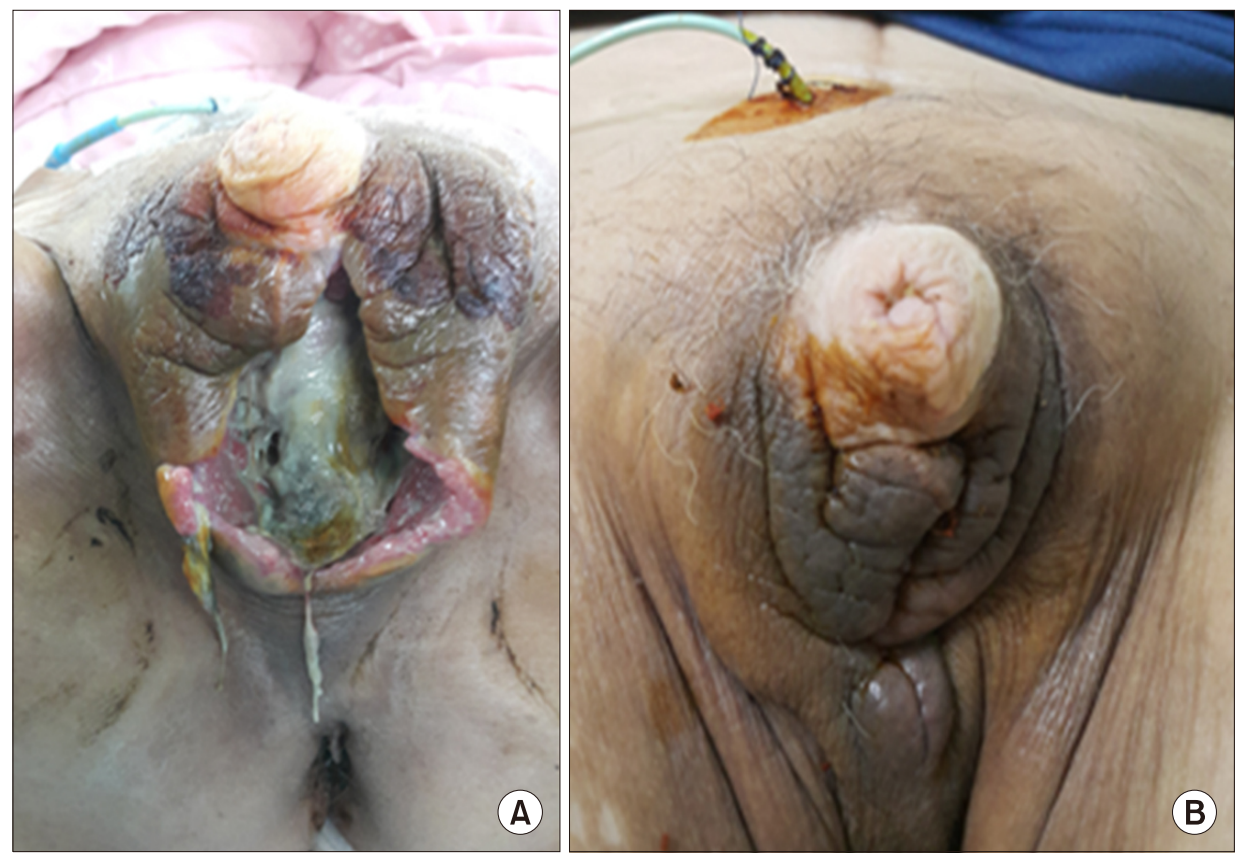

Fig. 2. (A, B) First incision and debridement. After discharge state. This study is a study that has been reviewed by the IRB.

Table 1. Antibiotics sensitivity of bacteria identified in the blood culture and pus culture

\begin{tabular}{|c|c|}
\hline \multicolumn{2}{|c|}{ Bacteria } \\
\hline Proteus mirabilis $^{\mathrm{a})}$ & Enterococcus faecalis ${ }^{\mathrm{b})}$ \\
\hline \multicolumn{2}{|l|}{ Result of sensitivity } \\
\hline Ampicillin: $S(\leq 2)$, & Ampicilli \\
\hline Amoxicillin/Clavulanic acid: & ulbactam: $S(\leq 2)$ \\
\hline $\begin{array}{l}\mathrm{S}(\leq 2) \\
\text { Amikarin. S (4) }\end{array}$ & Clindamycin: R $(\geq 8)$ \\
\hline $\begin{array}{l}\text { Amikacin: S }(4), \\
\text { Aztreonam: } S(\leq 1)\end{array}$ & $\begin{array}{l}\text { Ciprofloxacin: S (1), } \\
\text { Erythromycin: I (2), }\end{array}$ \\
\hline $\begin{array}{l}\text { Aztreonam: } S(\leq 1) \\
\text { Ciprofloxacin: } S(\leq 0.25) \text {, }\end{array}$ & $\begin{array}{l}\text { Erythromycin: I }(2), \\
\text { Nitrofurantoin: } S(\leq 16) \text {, }\end{array}$ \\
\hline Cefazolin: S $(\leq 4)$, & Gentamicin high level (synergy): \\
\hline Ertapenem: $\mathrm{S}(\leq 0.5)$, & S (SYN-S), \\
\hline Cefepime: S $(\leq 1)$ & Streptomycin high level \\
\hline Cefoxitin: S $(\leq 4)$ & (synergy): S (SYN-S), \\
\hline Gentamicin: $S(\leq 1)$, & Imipenem: S (2), \\
\hline Imipenem: R (4), & Levofloxacin: S (1), \\
\hline Trimethoprim/Sulfamethoxazole: & Linezolid: S (2), \\
\hline$S(\leq 20)$ & Norfloxacin: S (4), \\
\hline Cefotaxime: $\mathrm{S}(\leq 1)$, & Benzylpenicillin: S (8), \\
\hline Ceftazidime: $S(\leq 1)$, & Quinunristin/Dalfonristi \\
\hline Tigecycline: $\mathrm{R}(\leq 0.5)$, & Trimethoprim/Sulfamethoxazole: \\
\hline Piperacillin/ & $\mathrm{R}(\leq 1$ \\
\hline Tazobactam: S $(\leq 4)$ & Tetracycline: $R(\geq 16)$, \\
\hline & Tigecycline: $S(\leq 0.12)$, \\
\hline & $\begin{array}{l}\text { Teicoplanin: } S(\leq 0.5) \\
\text { Vancomycin: } S(1)\end{array}$ \\
\hline
\end{tabular}

${ }^{a}$ Blood culture, ${ }^{\text {b) }}$ pus culture.

training during the immediate post-operative period. On the other hand, artificial sphincter insertion is recommended if these methods are ineffective [5]. This operative therapy cannot be applied to all patients because of various etiologies. If so, many physicians might misuse an indwelling catheter to control the incontinence. This may induce vari- ous catheter-related problems, such as urinary tract infection, urethral stricture, and urethral rupture [6]. Therefore, The Canadian Agency for Drugs and Technologies in Health does not recommend the long-term use of a urethral catheter in patients with urinary incontinence [7].

For patients who have used indwelling catheters for long periods, it is necessary to check whether there is inflammation in the scrotum, even if there is no chronic disease such as diabetes. In addition, conservative management, including broad-spectrum antibiotic treatment and blood pressure control, should be considered.

Although the cause of the Fournier's gangrene was a perineal abscess, it may also occur when chronic catheterization is performed. Predisposing factors include diabetes mellitus, chronic renal failure, and congestive heart failure [1]. One research reported that the diagnosis time is crucial because it correlates directly to a person's death. The death rate of the surgical intervention within two days after hospitalization was significantly lower than the procedure delayed 3 to 5 days [8].

Although most of the causative bacteria of Fournier's gangrene are polymicrobial, Escherichia coli, Bacteroides spp., and Staphylococci, Proteus spp. are the main microorganisms. Other bacteria were identified in blood and wound cultures in this case. Nevertheless, research reported no correlation between the strain of microorganisms and mortality [9]. 
In conclusion, Fournier's gangrene requires urological emergency procedures, particularly for patients with the long-term use of a urethral catheter. These patients require careful physical examinations and immediate treatment to obtain good results.

\section{CONFLICT OF INTEREST}

No potential conflict of interest relevant to this article was reported.

\section{AUTHOR CONTRIBUTIONS}

K.K.P. participated in data collection and wrote the manuscript. S.D.K. and Y.J.K. participated in the study design and performed the statistical analysis. J.S.H. participated in the study design and coordination and helped to draft the manuscript. All authors read and approved the final manuscript.

\section{ORCID}

Kyung Kgi Park, https://orcid.org/0000-0001-9807-1461

Sung Dae Kim, https://orcid.org/0000-0002-0092-6060

Young-Joo Kim, https://orcid.org/0000-0001-5580-4338

Jung-Sik Huh, https://orcid.org/0000-0003-2767-4390

\section{REFERENCES}

1. Aridogan IA, Izol V, Abat D, Karsli O, Bayazit Y, Satar N.
Epidemiological characteristics of Fournier's gangrene: a report of 71 patients. Urol Int 2012;89:457-61.

2. Huh JS, Kim YJ, Kim SD, Park KK. The effectiveness of cystography-measured bladder neck elevation at predicting the return of continence after robot-assisted radical prostatectomy. Int Neurourol J 2019;23:234-9.

3. Karakiewicz PI, Tanguay S, Kattan MW, Elhilali MM, Aprikian AG. Erectile and urinary dysfunction after radical prostatectomy for prostate cancer in Quebec: a population-based study of 2415 men. Eur Urol 2004;46:188-94.

4. Hammerer $\mathrm{P}$, Huland $\mathrm{H}$. Urodynamic evaluation of changes in urinary control after radical retropubic prostatectomy. J Urol 1997;157:233-6.

5. Sandhu JS, Breyer B, Comiter C, Eastham JA, Gomez C, Kirages D), et al. Incontinence after prostate treatment: AUA/SUFU guideline. J Urol 2019;202:369-78.

6. Holroyd-Leduc JM, Sands LP, Counsell SR, Palmer RM, Kresevic DM, Landefeld CS. Risk factors for indwelling urinary catheterization among older hospitalized patients without a specific medical indication for catheterization. J Patient Saf 2005; 201-7.

7. Lachance CC, Grobelna A, editors. Management of patients with long-term indwelling urinary catheters: a review of guidelines. Ottawa: Canadian Agency for Drugs and Technologies in Health; 2019. p. 1-25.

8. Sugihara $T$, Yasunaga $H$, Horiguchi $H$, Fujimura $T$, Ohe $K$, Matsuda $\mathrm{S}$, et al. Impact of surgical intervention timing on the case fatality rate for Fournier's gangrene: an analysis of 379 cases. BJU Int 2012;110(11 Pt C):E1096-100.

9. Eke N. Fournier's gangrene: a review of 1726 cases. Br J Surg 2000;87:718-28. 